\title{
Entangled coherent states and squeezing in $N$ trapped ions
}

\author{
E. Solano, ${ }^{1,3}$ R. L. de Matos Filho, ${ }^{2}$ and N. Zagury ${ }^{2}$ \\ ${ }^{1}$ Max-Planck-Institut für Quantenoptik, Hans-Kopfermann-Strasse 1, 85748 Garching, Germany \\ ${ }^{2}$ Instituto de Física, Universidade Federal do Rio de Janeiro, Caixa Postal 68528, 21945-970 Rio de Janeiro, RJ, Brazil \\ ${ }^{3}$ Sección Física, Departamento de Ciencias, Pontificia Universidad Católica del Perú, Apartado 1761, Lima, Peru
}

(October 22, 2018)

\begin{abstract}
We consider a resonant bichromatic excitation of $N$ trapped ions that generates displacement and squeezing in their collective motion conditioned to their ionic internal state, producing eventually Schrödinger cat states and entangled squeezing. Furthermore, we study the case of tetrachromatic illumination for producing the so called entangled coherent states in two motional normal modes.
\end{abstract}

PACS number(s): 3.65.Ud, 42.50.Vk, 3.67.Hk

In the last years great attention has being given to the possibility of producing mesoscopic superposition of states where a great number of particles and degrees of freedom is involved [1] [4]. Beyond their possible applications in quantum information [5, [6], they represent an important tool in the experimental studies of decoherence and the emergence of the classical world out of its quantized version [7]. In the microscopic world, a system can exist in a superposition of different quantum states, given rise to interference effects. However, these superpositions do not manifest themselves in the classical world [8]. This paradox is partially solved by realizing that coherence is lost increasingly fast with the size of the system [2. 9]. Therefore, the generation of mesoscopic quantum superpositions, and the study of the time scale in which decoherence occurs, is an important step for understanding the boundary between classical physics and quantum mechanics. The system of $N$ trapped ions is a good laboratory for building large quantum superpositions and analyzing how decoherence appears when the system grows, since it is weakly affected by the environment [5.97. In this work, we discuss some proposals for producing, in a fast and controllable way, vibronic mesoscopic superpositions and other nonclassical states in a system consisting of $N$ trapped ions 10,11. In particular, generation of Schrödinger cat states, entangled squeezing and entangled coherent states are discussed.

We consider the situation where $N$ two-level ions of mass $m$ are confined to move in the $z$ direction of a Paul trap. We assume that they are cooled down to low temperatures 3.12] and able to perform small oscillations around their equilibrium positions, $z_{j 0}(j=1,2 \ldots N)$. Two classical fields, $\vec{E}_{I}=\vec{E}_{0 I} e^{i\left(q_{I} z-\omega_{I} t-\varphi_{I}\right)}$ and $\vec{E}_{I I}=$ $\vec{E}_{0 I I} e^{i\left(q_{I I} z-\omega_{I I} t-\varphi_{I I}\right)}$, illuminate homogeneously all $N$ ions, with angular frequencies $\omega_{I}$ and $\omega_{I I}$, wave vectors, $q_{I}=q_{I I}=q$, parallel to the $z$ direction, and phases $\varphi_{I}=\varphi_{I I}=\varphi$. Depending on the experimental setup, each of theses fields may be obtained effectively from a pair of lasers in Raman configuration. The angular frequencies $\omega_{I}$ and $\omega_{I I}$ are chosen to be quasiresonant with a ionic internal transition between long-living levels $\left|e_{j}\right\rangle$ and $\left|g_{j}\right\rangle(j=1, \ldots N)$, with energies $\hbar \omega_{0}$ and 0 , respec- tively. The total Hamiltonian of the system may be written, in the optical rotating wave approximation (RWA), as

$$
\hat{H}=\hat{H}_{0}+\hat{H}_{\text {int }},
$$

with

$$
\hat{H}_{0}=\hbar \omega_{0} \sum_{j=1, N}\left|e_{j}\right\rangle\left\langle e_{j}\right|+\hbar \nu a^{\dagger} a+\sum_{\lambda=1, N-1} \hbar \nu_{\lambda} b_{\lambda}^{\dagger} b_{\lambda},
$$

and

$$
\begin{gathered}
\hat{H}_{\mathrm{int}}=\hbar \Omega \sum_{j=1, N}\left[e^{i\left(q \hat{z}_{j}-i \omega_{I} t-\varphi\right)}+e^{i\left(q \hat{z}_{j}-i \omega_{I I} t-\varphi\right)}\right]\left|e_{j}\right\rangle\left\langle g_{j}\right| \\
+ \text { H.c. }
\end{gathered}
$$

The operators $\left\{a, b_{\lambda}\right\}$ and $\left\{a^{\dagger}, b_{\lambda}^{\dagger}\right\}$ are the annihilation and creation operators associated with the center-of-mass (CM) mode of frequency $\nu$ and with the $N-1$ other vibrational modes of frequency $\nu_{\lambda}$, respectively [13]. We assumed also that the same real coupling constant $\Omega$ appears in the interaction of each excitation field with each ion.

We choose the frequencies $\omega_{I}$ and $\omega_{I I}$ to be

$$
\omega_{I}=\omega_{0}+k \nu \quad \text { and } \quad \omega_{I I}=\omega_{0}-k \nu,
$$

so that we excite the CM vibronic transition in the $k$-th blue and $k$-th red motional sideband [14,15. We go to the interaction picture and make a RWA with respect to the CM vibrational frequency, selecting the terms that oscillate with minimum frequency [16]. For simplifying the notation, we set $\varphi=k \pi / 2$ and define the "spin angular momentum states" $\left|\uparrow_{j}\right\rangle=e^{i q z_{j 0}}\left|e_{j}\right\rangle$ and $\left|\downarrow_{j}\right\rangle=\left|g_{j}\right\rangle$. Then, in the Lamb-Dicke regime, the resulting effective Hamiltonian may be written as

$$
H_{\mathrm{int}}=\frac{\hbar \eta^{k} \Omega}{k !} \sum_{j=1}^{N}\left(\sigma_{j}^{\dagger}+\sigma_{j}\right)\left(a^{k}+a^{\dagger k}\right),
$$

where $\eta=q \sqrt{\hbar / 2 N m \nu}$ is the CM Lamb-Dicke parameter and $\widehat{\sigma}_{+j}=\left|\uparrow_{j}\right\rangle\left\langle\downarrow_{j}\left|=e^{i q z_{j 0}}\right| e_{j}\right\rangle\left\langle g_{j}\right|$ is the so redefined flipup operator. Note that, in the Hamiltonian of Eq. (4), 
the operators associated with the internal and external degrees of freedom are decoupled. In fact, it could be rewritten as

$$
H_{\mathrm{int}}=\frac{2 \hbar \eta^{k} \Omega}{k !} J_{x}\left(a^{k}+a^{\dagger k}\right)
$$

where $J_{x}=\sum_{j=1}^{N}\left(\sigma_{j}^{\dagger}+\sigma_{j}\right) / 2$ may be associated with the $x$ component of an angular momentum operator $\vec{J}$, being the $y$ and $z$ components $J_{y}=\sum_{j=1}^{N}\left(\sigma_{j}^{\dagger}-\sigma_{j}\right) /(2 i)$ and $J_{z}=\sum_{j=1}^{N}\left(\left|e_{j}\right\rangle\left\langle e_{j}|-| g_{j}\right\rangle\left\langle g_{j}\right|\right) / 2$, respectively. We now express the evolution operator, at time $t$, as a sum of products of unitary operators acting on the motional state and projection operators acting on the internal state of the ions

$$
U_{k}(t)=\sum_{j, m} D_{k}\left[m \Omega_{k} t\right]|j, m\rangle_{x x}\langle j, m|,
$$

with $\Omega_{k}=2 i \eta^{k} \Omega / k !$. Also,

$$
D_{k}(\chi)=e^{\chi a^{\dagger k}-\chi^{*} a^{k}}
$$

and $|j, m\rangle_{x}$ are the simultaneous eigenvectors of the operators $J_{x}$ and $J^{2} \equiv J_{x}^{2}+J_{y}^{2}+J_{z}^{2}$ associated with the eigenvalues $m=-j,-(j-1), \ldots \ldots j$ and $j(j+1)$, respectively. $j$ varies from $0(1 / 2)$ to $N / 2$ by steps of 1 , if $N$ is even (odd).

Eq. (6) shows an important feature of the proposed scheme, it yields an evolution that corresponds to unitary operations $\hat{D}_{k}(\chi)$ on the CM mode conditioned to the value $m$ of the $x$-component of the "angular momentum" electronic state. When $k=1$, the excitations occuring in the first red and first blue sidebands, Eq. (7) turns into the familiar displacement operator $D(\alpha)$, which produces the coherent state $|\alpha\rangle$ out of an initial vacuum state $|0\rangle$. For example, let us consider the case $N=1$ with an initial state $|\downarrow\rangle|0\rangle=(|+\rangle+|-\rangle) / \sqrt{2}$, where the sates $| \pm\rangle=(|\downarrow\rangle \pm|\uparrow\rangle) / \sqrt{2}$ are the eigenstates of the operator $J_{x}$ for the case of a single spin $1 / 2$. After a time $\tau$, the evolved state will be

$$
\frac{1}{\sqrt{2}}(|+\rangle|\alpha\rangle+|-\rangle|-\alpha\rangle),
$$

where $\alpha=i \eta \Omega \tau$. This state is usually called Schrödinger cat state, consisting of an entangled bipartite system correlating microscopic and mesoscopic quantum states. A measurement of the ionic internal state will project the motional state in

$$
\frac{1}{\sqrt{2}}(|\alpha\rangle \pm|-\alpha\rangle)
$$

usually called even and odd coherent states, if the outcome of the detection was $|\downarrow\rangle$ or $|\uparrow\rangle$, respectively. Bigger entangled and superposition states, involving different coherent states for the case of $N$ ions, were considered recently by the same authors 10.11].
The case $k=2$, the excitations occuring in the second red and second blue sidebands, can offer us other interesting nonclassical states. The operator of Eq. (7) turns into $S(\xi)$, a squeezing operator with squeezing parameter $\xi$, that creates two-photon coherent states [17]

$$
|\xi\rangle=e^{\left(\xi a^{\dagger 2}-\xi^{*} a^{2}\right)}|0\rangle .
$$

If, again, we choose $N=1$ (for $k=2$ ) and an initial state $|\downarrow\rangle|0\rangle=(|+\rangle+|-\rangle) / \sqrt{2}$, the final state after an interaction time $\tau$ is

$$
\frac{1}{\sqrt{2}}(|+\rangle|\xi\rangle+|-\rangle|-\xi\rangle)
$$

where $|\xi\rangle$ and $|-\xi\rangle$ are squeezed vacuum states with $\xi=i \eta^{2} \Omega / 2$. These squeezing parameters, $\xi=r e^{i \phi}$ and $-\xi=r e^{i(\phi+\pi)}$, will produce the same compression $e^{-r}$ in the motional quadratures associated with the orthogonal directions $\phi / 2$ and $\phi / 2+\pi / 2$, respectively. The state of Eq. (11) is, then, an entangled state correlating the microscopic states $| \pm\rangle$ with the orthogonally squeezed vacuum states $| \pm \xi\rangle$, produced with a single bichromatic Raman laser pulse. When comparing the entangled states of Eq. (\&) and those of Eq. (11), note that coherent states are considered as "classical" states and squeezed states as "nonclassical" ones 18. Going to higher values of $N$ will produce, as can be seen from Eq. (6), multiple entanglement of different microscopic internal states with different squeezed vacuum states. Note, also, that it is possible to produce squeezing in a chosen motional quadrature without affecting or entangling the internal degrees of freedom [19]. For achieving this goal, it is enough to consider an initial state that contains one of the electronic eigenstates, say $|j, m\rangle_{x}|0\rangle$, that in the case of the previous example $(N=1)$ can be written as $| \pm\rangle|0\rangle$.

We now consider the simultaneous application of four Raman laser fields on $N$ trapped ions $(N>2)$. Their frequencies are chosen as

$$
\begin{aligned}
\omega_{I} & =\omega_{0}+k \nu, \quad \omega_{I I}=\omega_{0}-k \nu, \\
\omega_{I I I} & =\omega_{0}+k \nu_{r}, \quad \omega_{I V}=\omega_{0}-k \nu_{r},
\end{aligned}
$$

where $\nu_{r}=\sqrt{3} \nu$ is the frequency associated with the stretch vibrational mode. By so doing, we excite simultaneously the $k$-th red and the $k$-th blue vibronic sidebands of the CM and the stretch mode. The amplitudes of the fields exciting the CM mode are taken as equal and the ones exciting the stretch mode follow a similar condition. We take the phases of all Raman laser pulses equal to $\varphi=k \pi / 2$. The effective Hamiltonian, after following the same steps as in the case of the bichromatic illumination, reads

$$
H_{\mathrm{int}}=\frac{2 \hbar}{k !} J_{x}\left[\eta^{k} \Omega\left(a^{k}+a^{\dagger k}\right)+\eta_{r}^{k} \Omega_{r}\left(b^{k}+b^{\dagger k}\right)\right],
$$

where $b$ and $b^{\dagger}$ are the annihilation and creation operator of the stretch mode and $\eta_{r}=\eta / \sqrt[4]{3}$ its associated 
Lamb-Dicke parameter. $\Omega$ and $\Omega_{r}$ are the different coupling constants associated with the $\mathrm{CM}$ and stretch mode excitations, respectively. Using a similar reasoning, it is easy to see that the time evolution operator is now given by

$$
U(t)=\sum_{j, m} D_{k}\left(m \Omega_{k} t\right) D_{k r}\left(m \Omega_{k r} t\right)|j, m\rangle_{x}\langle j, m| .
$$

Here, $\Omega_{k r}=2 i \eta_{r}^{k} \Omega_{r} / k !$ and

$$
D_{k r}\left(\chi_{r}\right)=e^{\chi_{r} b^{\dagger k}-\chi_{r}^{*} b^{k}} .
$$

In what follows we will restrict us to the case $k=1$. We can say, then, that Eq. (14) shows the possibility of producing simultaneous displacements in the CM and stretch mode conditioned to the collective internal state.

Let us consider the particular case $N=2$ and an initial state $|\downarrow \downarrow\rangle|00\rangle=\frac{1}{2}\left(\left|\phi_{1}\right\rangle+\left|\phi_{2}\right\rangle+\left|\phi_{3}\right\rangle+\left|\phi_{4}\right\rangle\right)|00\rangle$, where

$$
\begin{aligned}
& \left|\phi_{1,2}\right\rangle=\frac{1}{2}(|\downarrow \downarrow\rangle \pm|\downarrow \uparrow\rangle \pm|\uparrow \downarrow\rangle+|\uparrow \uparrow\rangle) \\
& \left|\phi_{3,4}\right\rangle=\frac{1}{2}(|\downarrow \downarrow\rangle \pm|\downarrow \uparrow\rangle \mp|\uparrow \downarrow\rangle-|\uparrow \uparrow\rangle)
\end{aligned}
$$

are the eigenvectors of $J_{x}=\sum_{j=1}^{2}\left(\sigma_{j}^{\dagger}+\sigma_{j}\right) / 2(\operatorname{spin} 1)$ with eigenvalues $\lambda_{1,2}= \pm 1$ and $\lambda_{3,4}=0$, respectively. The application of the four Raman laser fields during a time $\tau$ will produce the entangled state

$$
\frac{1}{2}\left(\left|\phi_{1}\right\rangle|\alpha, \beta\rangle+\left|\phi_{2}\right\rangle|-\alpha,-\beta\rangle+\left|\phi_{3}\right\rangle|00\rangle+\left|\phi_{4}\right\rangle|00\rangle\right),
$$

with $\alpha=2 i \eta \Omega \tau$ and $\beta=2 i \eta_{r} \Omega_{r} \tau$. This is a more sophisticated Schrödinger cat state, correlating several microscopic states with the vacuum and different mesoscopic states in the CM and stretch motional modes. Measuring the internal state $|\downarrow \uparrow\rangle$ or $|\uparrow \downarrow\rangle$ will project the motional state into

$$
\frac{|\alpha, \beta\rangle-|-\alpha,-\beta\rangle}{\sqrt{2\left(1-e^{\left.-2|\alpha|^{2}-2|\beta|^{2}\right)}\right.}}
$$

These states, called entangled coherent states, own special entanglement properties [20], and have found diverse applications in the domain of quantum information theory 21-23]. In the language of the Gedankenexperiment after Schrödinger [8], the state of Eq. (18) represents the simultaneous occurrence of two alife cats and two dead cats. To our knowledge it is the first proposal for producing such entangled states in normal modes of $N$ trapped ions, with homogeneous illumination, involving quantum mechanical correlations between two classical states [24. It is clear that, by imposing $\eta \Omega=\eta_{r} \Omega_{r}$, we could have made $\alpha=\beta$. If in Eq. (17) we measure $|\downarrow \downarrow\rangle$ or $|\uparrow \uparrow\rangle$, we will project the motional state into

$$
\mathcal{N}(|\alpha, \beta\rangle+|-\alpha,-\beta\rangle \pm 2|00\rangle),
$$

respectively, where $\mathcal{N}$ is a normalization constant. A more elaborated way of producing entangled coherent states is to consider the initial state $\frac{1}{\sqrt{2}}(|\downarrow \downarrow\rangle+|\uparrow \uparrow\rangle)=$ $\frac{1}{\sqrt{2}}\left(\left|\phi_{1}\right\rangle+\left|\phi_{2}\right\rangle\right)$ [25.26. The application of the four Raman laser fields during a time $\tau$ generates the state

$$
\frac{1}{\sqrt{2}}\left(\left|\phi_{1}\right\rangle|\alpha, \beta\rangle+\left|\phi_{2}\right\rangle|-\alpha,-\beta\rangle\right) .
$$

The subsequent detection of the state $|\downarrow \downarrow\rangle$ will produce the state

$$
\frac{|\alpha, \beta\rangle+|-\alpha,-\beta\rangle}{\sqrt{2\left(1+e^{\left.-2|\alpha|^{2}-2|\beta|^{2}\right)}\right.}}
$$

and the measurement of the state $|\uparrow \uparrow\rangle$ will reproduce the entangled coherent state of Eq. (18).

It is easy to generalize this procedure to $n$ vibrational normal modes in an $N$ ion system, by using different bichromatic excitation fields with frequencies $\omega_{o} \pm k \nu_{n}, \nu_{n}$ being the frequency of the $n^{\text {th }}$ normal mode. By so doing, we could obtain more sophisticated entangled mesoscopic superpositions.

In conclusion, we have shown that resonant bichromatic and tetrachromatic excitations of $N$ trapped ions can be an important tool for producing different families of nonclassical states, involving the ionic internal and external degrees of freedom. Generating bigger nonclassical states, and studying their decay properties, should help us to understand better the border between the quantum and the classical world. At the same time, interesting applications may appear, specially the ones related to the field of quantum information. In particular, for the case of a single ion and a bichromatic illumination, we have shown how to produce Schrödinger cat states and entangled squeezed states by means of a single bichromatic Raman laser pulse. In the case of two ions and tetrachromatic excitation, we have shown how to produce, in a straightforward manner, entangled coherent states in two motional normal modes, and other states involving multiple entanglement.

This work was partially supported by the Conselho Nacional de Desenvolvimento Científico e Tecnológico (CNPq), the Fundação de Amparo a Pesquisa do Estado do Rio de Janeiro (FAPERJ), the Programa de Apoio a Núcleos de Excelência (PRONEX) and Fundação José Bonifácio (FUJB).

[1] C. Monroe, D. M. Meekhof, B. E. King, and D. J. Wineland, Science 272, 1131 (1996).

[2] M. Brune, E. Hagley, J. Dreye, X. Maître, A. Maali, C. Wunderlich, J. M. Raimond, and S. Haroche, Phys. Rev. Lett. 77, 4887 (1996). 
[3] C. A. Sackett, D. Kielpinski, B. E. King, C. Langer, V. Meyer, C. J. Myatt, M. Rowe, Q. A. Turchette, W. M. Itano, D. J. Wineland and C. Monroe, Nature 404, 256 (2000).

[4] A. Rauschenbeutel, G. Nogues, S. Osnaghi, P. Bertet, M. Brune, J. M. Raimond, and S. Haroche, Science 288, 2024 (2000).

[5] D. J. Wineland, C. Monroe, W. M. Itano, D. Leibfried, B. E. King, D. M. Meekhof, J. Res. NIST 103, 259-328 (1998).

[6] J. M. Raimond, M. Brune, and S. Haroche, Rev. Mod. Phys. 73, 565 (2001).

[7] See, for example, J. P. Paz and W. H. Zurek in Coherent Matter Waves, Proceedings of Les Houches Summer School, Session LXXII, edited by R. Kaiser, C. Westbrook, and F. David (EDP Sciences, Les Ulis; SpringerVerlag, Berlin, 2001).

[8] E. Schrödinger, Naturwissenschaften 23, 807(1935).

[9] C. J. Myatt, B. E. King, Q. A. Turchette, C. A. Sackett, D. Kielpinski, W. M. Itano, C. Monroe, and D. J. Wineland, Nature 403, 269 (2000).

[10] E. Solano, R. L. de Matos Filho, and N. Zagury, Phys. Rev. Lett. 87, 060402 (2001).

[11] E. Solano, R. L. de Matos Filho, and N. Zagury, Lecture Notes in Physics, 2001, no. 575, pp. 14-28, SpringerVerlag.

[12] B. E. King, C. S. Wood, C. J. Myatt, Q. A. Turchette, D. Leibfried, W. M. Itano, C. Monroe, and D. J. Wineland, Phys. Rev. Lett. 81, 1525 (1998).

[13] D. F. V. James, Appl. Phys. B 66, 181 (1998).

[14] S. Wallentowitz and W. Vogel, Phys. Rev. A 54, 3322 (1996).

[15] H. Moya-Cessa, S. Wallentowitz, and W. Vogel, Phys. Rev. A 59, 2920 (1999).

[16] W. Vogel and R.L. de Matos Filho, Phys. Rev. A52, 4214 (1995).

[17] H. P. Yuen, Phys. Rev. A13, 2226 (1971).

[18] S.-C. Gou, J. Steinbach, and P. L. Knight, Phys. Rev. A 55, 3719 (1997).

[19] D. M. Meekhof, C. Monroe, B. E. King, W. M. Itano, and D. J. Wineland, Phys. Rev. Lett. 76, 1796 (1996).

[20] O. Hirota and M. Sasaki, LANL e-print quant$\mathrm{ph} / 0101018$.

[21] S. J. van Enk and O. Hirota, Phys. Rev. A 64, 022313 (2001).

[22] W. J. Munro, G. J. Milburn, and B. C. Sanders, Phys. Rev. A 62, 052108 (2000).

[23] H. Jeong and M. S. Kim, LANL e-print quant$\mathrm{ph} / 0111015$.

[24] J. Steinbach, J. Twamley, and P. L. Knight, Phys. Rev. A 56, 4815 (1997).

[25] A. Sørensen and K. Mølmer, Phys. Rev. Lett. 82, 1971 (1999).

[26] E. Solano, R. L. de Matos Filho, and N. Zagury, Phys. Rev. A, 59, R2539 (1999); 61, 029903(E) (2000). 